\title{
Comparison theorems for supremum norms
}

\author{
By
}

HANS SCHNEIDER * and W. GILBERT STRANG

1

The problem of characterizing all supremum norms on a space of matrices or linear transformations is still unsolved. The theorems of this note are intended as a step towards solving this problem. Our most general result is Theorem 3, in which the assumption of finite dimensionality is not needed. Theorems 1 and 2 are special cases of Theorem 3. In view of the independent interest of Theorem 1, we have thought it desirable to include a separate proof of this case.

2

In this note all vector spaces $V$ considered will be over the real or complex numbers. By a norm $v$ on $V$ we shall mean an equilibrated norm; thus $v$ maps $V$ into the nonnegative numbers and, if $x, y \in V$ and $c$ is real or complex, then

$$
\begin{gathered}
\nu(x)>0 \quad \text { if } \quad x \doteqdot 0 \\
\nu(x+y) \leqq \nu(x) \div v(y), \\
\nu(c x)=|c| \nu(x) .
\end{gathered}
$$

Let $U$ be a vector space normed by $\mu$ and $V$ a vector space normed by $\nu$. If $T$ is a space of transformations of $U$ into $Y$, for which $\frac{\nu(A x)}{\mu(x)}$ is bounded over
$x$ in $U$ for all $A$ in $T$, then sup defined by

$$
\sup (A)=\sup _{0 \neq x \in U} \frac{\nu(A x)}{\mu(x)}, \quad A \in T
$$

is a norm on $T$. In this case it is natural to call sup the supremum norm belonging to $(\mu, v)$. If $U=V$ and $\mu=v$, we call sup the supremum norm belonging to the single norm $\nu$. A special case of a supremum norm is the dual norm $\nu^{\prime}$ of $v$ on the dual space $V^{\prime}$ of $V$. Here $V^{\prime}$ consists of all bounded linear functionals on $V$. Thus

$$
\nu^{\prime}\left(y^{\prime}\right)=\sup _{0 \neq x \in V} \frac{\left|\left(y^{\prime}, x\right)\right|}{y^{\prime}(x)}, \quad y^{\prime} \in V^{\prime}
$$

If $V$ is identified with a subspace of $V^{\prime \prime}$ in the canonical way, then it is known (e.g. Householder [2]) that $\nu^{\prime \prime}(x)=v(x)$, for all $x \in V$. This result will be used

* The research of first named author was done while he was a summer participant at Oak Ridge National Laboratory, Oak Ridge, Tennessee. 
in the proof of Theorem 1. We shall also use a result which applies to finite dimensional spaces only and which is a consequence of the conpactness of the unit ball $\{x: \nu(x) \leqq 1\}$ in these spaces: say

If $\nu_{1}$ and $\nu_{2}$ are norms on the finite dimensional space $V$, then $\frac{\nu_{1}}{\nu_{2}}$ is bounded,

$$
\sup _{v} \frac{y_{1}}{y_{2}}=n
$$

and the equality is attained [3]; i.e., for some $v \in V$,

$$
\frac{v_{1}(v)}{v_{2}(v)}=n
$$

In equation (6) we used the notational convention of writing $\sup _{V} \frac{\nu_{1}}{\nu_{2}}$ to mean $\sup _{0 \neq x \in V} \frac{\nu_{1}(x)}{\nu_{2}(x)}$. Similarly we shall write $\nu_{1} \leqq \nu_{2}$ to mean $\nu_{1}(x) \leqq \nu_{2}(x)^{V}$ for all $x \in V$.

3

Theorem 1. Let $V$ be a finite dimensional vector space. Let $v_{1}$ and $v_{2}$ be nornus on $V$, and let $\sup _{i}, i=1,2$, be the norm on the space $L$ of all linear transformations of $V$ into itself belonging to the single norm $v_{i}$. If

then actually

$$
\begin{aligned}
\sup _{1} \leqq \sup _{2} & \sup _{1}=\sup _{2} \\
\nu_{1} & =n v_{2} .
\end{aligned}
$$

Proof. Let $n$, be defined by (6) and suppose that $v$ satisfies (7). It is an immediate consequence of (6) and of the definition (5) of the dual norm that

$$
y_{2}^{\prime} \leqq n \nu_{1}^{\prime} .
$$

Under the hypothesis (8), we shall prove the reverse inequality. For every $y^{\prime} \in V^{\prime}$ we may define the projection $E$ on $V$ by

$$
E x=\left(y^{\prime}, x\right) v,
$$

for all $x \in V$. By (3) and (12) we have

whence by (5)

$$
\sup _{0 \neq x \in V} \frac{\nu_{1}(E x)}{\nu_{1}(x)}=\sup _{0 \neq x \in V} \frac{\left|\left(y^{\prime}, x\right)\right|}{\nu_{1}(x)} \nu_{1}(v)
$$

$$
\sup _{1}(E)=\nu_{1}^{\prime}\left(y^{\prime}\right) \nu_{1}(v),
$$

and similarly

$$
\sup _{2}(E)=\nu_{2}^{\prime}\left(y^{\prime}\right) \nu_{2}(v) .
$$

Hence the hypothesis (8) and equation (7) imply

$$
n v_{1}^{\prime} \leqq v_{2}^{\prime} \text {. }
$$


Thus $n v_{1}^{\prime}=\gamma_{2}^{\prime}$, by (11) and (16), whence $\nu_{1}^{\prime \prime}=n v_{2}^{\prime \prime}$, and therefore (10) follows by the duality theorem $v^{\prime \prime}(x)=v(x)$, for $x \in V$. The equality (9) is an immediate consequence of (10).

\section{4}

Our first generalization of Theorem 1 is

Theorem 2. Let $V$ and $L$ be as defined in Theorem 1. If $\sup _{i}, i=1,2$, is

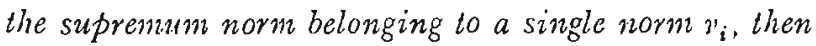

$$
\text { - } \quad \inf _{L} \frac{\sup _{2}}{\sup _{2}}=\inf _{L} \frac{\sup _{2}}{\sup _{1}} \text {. }
$$

Theorem 2 is clearly a consequence of Theorem 3 below. For, let $U$ and $V$ be vector spaces normed by $\mu_{i}, y_{i}, i=1,2$, and suppose

$$
m_{1}=\inf _{U} \frac{\mu_{2}}{\mu_{1}}, \quad m_{2}=\inf _{U} \frac{\mu_{1}}{\mu_{2}}, \quad n_{1}=\inf _{V} \frac{\nu_{2}}{\nu_{1}}, \quad n_{2}=\inf _{V} \frac{\nu_{1}}{v_{2}} .
$$

If we put $U=V$ and $\mu_{i}=\nu_{i}, i=1,2$, then $m_{1}=n_{1}, m_{2}=n_{2}$. Hence $m_{1} n_{2}=n_{2} n_{1}$ and Theorem 2 follows from Theorem 3. Of course, if $U$ and $V$ are finite dimensional, then $m_{i}$ and $n_{i}$ are non-zero, and it will be convenient to assume this for the infinite-dimensional case which follows. Thus the norms will be topologically equivalent, and a transformation bounded with respect to $\mu_{1}, \nu_{1}$ is bounded with respect to $\mu_{2}, \nu_{2}$, and vice versa.

5

Theorem 3. Let $U$ and $V$ be vector spaces (possibly infinite dimensional), and let $\mu_{i}, \nu_{i}, i=1,2$, be norms on $U$ and $V$ respectively, and let $m_{1}, n_{2}$ be defined by (18) and be non-zero. Let $T$ be a set of bounded transformations which contains all bounded linear transformations of rank 1 . Let $\sup _{1}$ and $\sup _{2}$ be the norms on $T$ belonging to $\mu_{1}, \nu_{1}$, and $\mu_{2}, \nu_{2}$. Then

$$
\inf _{\Upsilon} \frac{\sup _{1}}{\sup _{2}}=m_{1} n_{2} \text {. }
$$

Proof. Let $A \in T$, and $x \in U$. Then, if $A x \neq 0$,

$$
\frac{\nu_{1}(A x)}{\mu_{1}(x)}=\frac{\mu_{2}(x)}{\mu_{1}(x)} \frac{v_{1}(A x)}{v_{2}(A x)} \frac{v_{2}(A x)}{\mu_{2}(x)} \geqq m_{1} n_{2} \frac{v_{2}(A x)}{\mu_{2}(x)},
$$

and, since this inequality is trivial if $A x=0$, we have *

$$
\frac{\sup _{1}}{\sup _{2}} \geqq m_{1} n_{2} \text {. }
$$

Let $m$ and $n$ be any positive numbers which satisfy

$$
m_{1}<m, \quad n_{2}<n .
$$

* The argument leading to (21) is equivalent to a remark of HouseHordeR's [2], Chapter 2. We are grateful to Dr HouseHolder for permitting us to see his book in manuscript form.

Numer. Math. Bd. 4 
To complete the proof of the theorem, we shall demonstrate the existence of a linear transformation $E$ of $U$ into $V$ of rank 1 for which

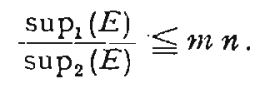

In virtue of the definitions (18) there exist $\rightsquigarrow \in U, v \in Y$ such that

$$
\frac{\mu_{2}(u)}{\mu_{2}(u)} \leqq m, \quad \frac{v_{1}(v)}{r_{2}\left(v^{\prime}\right)} \leqq n
$$

On the one dimensional subspace $\{u\}$ we define a linear functional $y_{0}^{\prime}$ by

$$
\left|\left(y_{0}^{\prime}, x\right)\right|=\mu_{1}(x) \mu_{2}(u), \quad x \in\{u\} .
$$

It follows by the Hahn-Banach Theorem ([1], p. 11) that there exists an extension linear functional $y^{\prime}$ of $y_{0}^{\prime}$ for which

$$
\left|\left(y^{\prime}, x\right)\right| \leqq \mu_{1}(x) \mu_{2}(u),
$$

for all $x \in U$. If we now define $E$ by

$$
E x=\left(y^{\prime}, x\right) v,
$$

we obtain immediately that for all $x \in U$

whence

$$
\frac{v_{2}(E x)}{\mu_{1}(x)}=\frac{\left|\left(y^{\prime}, x\right)\right| v_{1}(v)}{\mu_{1}(x)} \leqq \mu_{2}(u) \nu_{1}(v),
$$

$$
\sup _{1}(E) \leqq \mu_{2}(u) \nu_{1}(v)
$$

But

whence

$$
\frac{v_{2}(E u)}{\mu_{2}(u)}=\frac{\left|\left(y^{\prime}, u\right)\right| v_{2}(v)}{\mu_{2}(u)}=\mu_{1}(u) u_{2}(v)
$$

$$
\sup _{2}(E) \geqq \mu_{1}(u) \gamma_{2}(v) .
$$

The inequality (23) now follows by (24) and the theorem is proved.

6

The following corollary includes assertion (10) of Theorem 1.

Corollary. If $\frac{\sup _{1}}{\sup _{2}}$ is a constant on $T$, then $\frac{\mu_{2}}{\mu_{1}}$ and $\frac{v_{1}}{v_{2}}$ are constants on $U$ and $V$ (and, trivially, conversely).

Proof. By the definitions (18)

$$
n_{1} n_{2} \leqq 1, \quad n_{1} n_{2} \leqq 1,
$$

and $\frac{\mu_{1}}{\mu_{2}}, \frac{\nu_{1}}{\nu_{2}}$ are constant if and only if

$$
m_{1} m_{2}=1, \quad n_{1} n_{2}=1
$$


But, in virtue of (19), $\frac{\sup _{1}}{\sup _{2}}$ is a constant if and only if

$$
\frac{\sup _{1}}{\sup _{2}}=m_{1} n_{2}, \quad m_{2} n_{1}=\frac{\sup _{2}}{\sup _{1}}=\frac{1}{m_{1} n_{2}} .
$$

Hence $\frac{\sup _{1}}{\sup _{2}}$ is a constant if and only if

$$
\left(m_{1} m_{2}\right)\left(n_{1} n_{2}\right)=1
$$

and so, by (32), if and only if (33) holds. The corollary is proved.

We give now a geometric version of the above proof, assuming for the sake of clarity that $\frac{\mu_{2}}{\mu_{1}}$ and $\frac{\nu_{1}}{\nu_{2}}$ attain their infima, say

$$
\frac{\mu_{2}(u)}{\mu_{1}(u)}=m_{1}, \quad \frac{v_{1}(v)}{v_{2}(v)}=n_{2} .
$$

Suppose $M_{i}, N_{i}$ are the convex bodies belonging to the norms $\mu_{i}, v_{i}: M_{i}=$ $\left\{x \in U: \mu_{i}(x) \leqq 1\right\}$. We may choose $u$ and $v$ satisfying (36) on the boundaries $M_{1}^{0}, N_{2}^{0}$ of $M_{1}, N_{2}$ respectively. Let $u+H$ be the support plane of $M_{1}$ at $u$, where $H$ is a maximal subspace of $U$. Then the linear transformation $E$ defined by $E u=v, E H=0$, has $\frac{\sup _{1}(E)}{\sup _{2}(E)}=n_{1} n_{2}$. This construction is in fact analogous to the construction of $E$ in the proof of Theorem 3. We shall only remark here that the Hahn-Banach Theorem implies the existence of a support plane for $M_{1}$ at every point of $M_{1}^{0}$ : for, ${ }^{\star}$ if $u \in M_{1}^{0}$ and $y^{\prime}$ satisfies (25) and (26), then $u+H=\left[x \in U:\left(y^{\prime}, x\right)=\mu_{2}(u)\right\}$ is a support plane for $M_{1}$ at $u$.

We shall also relate the proof of Theorem 1 to the proof of the more general Theorem 3. As already indicated, the proof of Theorem 1 depends on the theorem that $v^{\prime \prime}(x)=v(x)$, for all $x \in V$, under the natural identification of $V$ with a subspace of $V^{\prime \prime}$. Now, if $x \in V$, then

$$
\nu^{\prime \prime}(x)=\sup _{y^{\prime}\left(y^{\prime}\right)=1}\left|\left(y^{\prime}, x\right)\right|
$$

and if $\nu^{\prime}\left(y^{\prime}\right)=1$ then $\left|\left(y^{\prime}, x\right)\right| \leqq \nu(x)$ by (5). Hence $\nu^{\prime \prime}(x)=\nu(x)$ if there exists $y^{\prime} \in V^{\prime}$ such that $\left|\left(y^{\prime}, x\right)\right|=\nu(x)$ and $\left|\left(y^{\prime}, z\right)\right| \leqq \nu(z)$, for all $z \in V$. Thus the duality theorem is implied by the Hahn-Banach Theorem for one-dimensional subspaces.

8

Theorem 1, and therefore, of course, Theorems 2 and 3, are false for nonequilibrated norms $\mu_{i}, \boldsymbol{v}_{i}$, viz. those for which (3) is replaced by

$$
\nu(c x)=c \nu(x), \quad \text { if } \quad c>0 .
$$

$\star$ We are grateful to Professor Wr. Rudin for this remark. 
20 H. Schneider and W.G. Strang: Comparison theorems for supremum norms

For let $V$ be the real line, and let

but let

$$
\nu_{1}(x)=v_{2}(x)=x \quad \text { if } \quad x \geqq 0,
$$

$$
v_{1}(x)=2 v_{2}(x)=-x \quad \text { if } \quad x<0 .
$$

If $r$ is identified with the linear transformation $x \rightarrow r x$ then

but

$$
\sup _{1}(r)=\sup _{2}(r)=r \quad \text { if } \quad r>0,
$$

$$
\sup _{2}(r)=2 \sup _{1}(r)=-2 r \quad \text { if } \quad r<0
$$

Hence $\sup _{2} \geqq \sup _{1}$, but $\sup _{2} \neq \sup _{1}$.

Finally we remark that a result slightly more general" than theorem 3 may be proved. If the set $T$ contains all transformations of rank 1 then the assumption that $m_{i}, n_{i}, i=1,2$, are non-zero is not needed, and theorem 3 can be formulated as a theorem on quasi-norms.

\section{References}

[1] DAy, M. M. : Nomned Linear Spaces. Ergebnisse der Mathematik und ihrer Grenzgebiete, N. F., Heft 21. Berlin-Göttingen-Heidelberg: Springer 1958.

[2] Householder, A. S.: The theory of matrices in numerical analysis. (Unpublished.)

[3] Ostrowski, A.: Úber Normen von Matrizen. Math. Z. 63, 2-18 (1955).

$$
\begin{gathered}
\text { Mathematics Department } \\
\text { University of Wisconsin } \\
\text { Madison, Wisconsin } \\
\text { and } \\
\text { Mathematical Institute } \\
\text { Oxford } \\
10 \text { Parks Road }
\end{gathered}
$$

(Received September 18, 1961) 